\title{
Targeted Amplicon Sequencing in Genetic Diagnostics of Patients with Cystic Fibrosis
}

\author{
J.Kusic-Tisma ${ }^{1}$, A. Divac ${ }^{1}$, M. Ljujic ${ }^{1}$, N. Pejanovic ${ }^{1}$, B. Stanic ${ }^{1}$, D. Radojkovic ${ }^{1}$
}

${ }^{1}$ Institute of Molecular Genetic and Genetic Engineering, Belgrade, Serbia

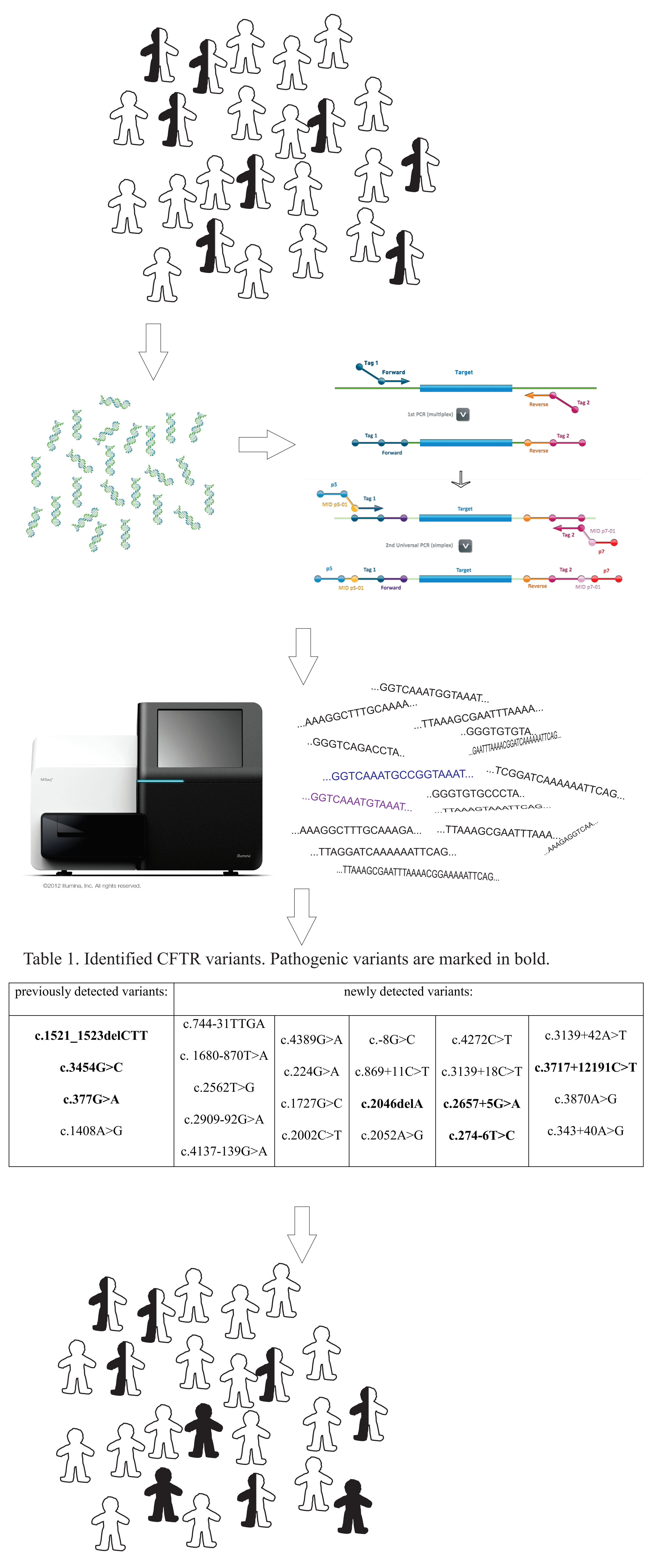

\section{Introduction}

Cystic fibrosis (CF, MIM \# 219700), one of the most common life-threatening autosomal recessive genetic disorders in Caucasians, is caused by mutations in the gene encoding the $\mathrm{CF}$ transmembrane conductance regulator (CFTR, MIM\# 602421). Molecular diagnosis of CF is essential for confirmation of clinical diagnosis and establishment of genetic counselling, prenatal diagnosis, cascade screening in families, as well as understanding the genotype-phenotype relationship. So far, almost 2000 CFTR gene variants with variable clinical significance have been identified, making the genetic diagnosis of CF difficult.

In this study we performed targeted resequencing of CFTR gene in $24 \mathrm{CF}$ patients in which one or no mutations were identified after analysis of 7 most common variants (c.1521_1523delCTT, c.489+1G $>$ T, c.1624G $>$ T,

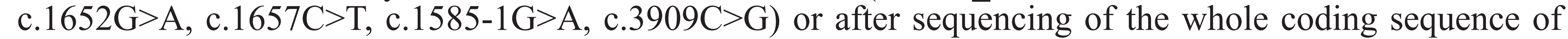
CFTR gene.

\section{Methods}

Targeted resequencing was performed using CFTR MASTR kit (Multiplicom). The technology is based on multiplex amplification of coding regions of the CFTR gene and selected intronic variants. The resulting amplicon library was pair-end sequenced on a MiSeq system (Illumina Inc., San Diego, CA) using MiSeq Reagent Kit v2 (500 cycles). Reliability of base call was demonstrated by average coverage greater than 1000 and the fact that 95.3 percent of bases has the Quality Score equal or above 30.

Fastq files produced upon library sequencing were processed by Sequencing Pilot software (JSI Medical Systems). Data Analysis included trimming of the PCR primer sequences from the reads. Evaluation of detected variants for disease relevance was based on the CFTR databases: http://www.genet.sickkids.on.ca/app and http://www.cftr2.org. All pathogenic sequence changes were confirmed by Sanger sequencing.

\section{Results}

The NGS sequencing data correctly confirmed all four germline variants previously detected in analyzed patients (Table 1.). Out of 21 additionally identified variants, four were classified as disease-causing mutations according to the literature data, hence enabling us confirmation of clinical CF diagnosis in 3 patients.

\section{Conclusion}

The NGS technology in combination with a well-characterized clinically relevant genomic variation database is a good alternative for a time consuming stepwise testing of genes with large allelic heterogeneity such as CFTR. 